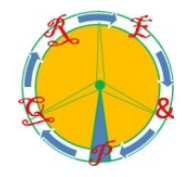

\title{
Optimal Voltage Sags Monitoring Considering Different Loading Profiles in Distribution Systems
}

\author{
T. R. Kempner ${ }^{1}$, F. A. Mourinho ${ }^{1}$, F. B. Bottura ${ }^{1}$, M. Oleskovicz ${ }^{1}$, J. C. M. Vieira ${ }^{1}$ and J. R. Lima Filho ${ }^{2}$ \\ ${ }^{1}$ Department of Electrical and Computer Engineering \\ University of São Paulo - São Carlos School of Engineering \\ Laboratório de Sistemas de Energia Elétrica - 400 Trabalhador São-carlense Avenue - São Carlos, SP, (Brazil) \\ Phone: +55 1633738149 , e-mail: kempner@.usp.br, mourinhofabricio@usp.br, fernando.bottura@usp.br, olesk@sc.usp.br, \\ jcarlos@sc.usp.br \\ ${ }^{2}$ Research, Development and Energetic Efficiency Managment \\ Eletrobras Distribuição Piauí - EDPI \\ 759 Maranhão Avenue - Teresina, PI, Brazil \\ Phone: +55 86 32288010, e-mail: ribamarlima@eletrobraspiaui.com
}

\begin{abstract}
This research aims to assess the influence of different distribution systems loading profiles on the optimized placement process of Power Quality (PQ) monitors. Therefore it is presented a placement methodology that considers different loading profiles in the IEEE 34 nodes test feeder. The placement methodology is a branch and bound based algorithm which includes single phase short-circuits simulations with different fault resistances at each one of all considered loading profiles. An observability index is evaluated and introduced in the optimization process in order to prioritize monitors placement at the most sensitized nodes regarding voltage sags. Results reveal that the proposed placement methodology was able to provide feasible solutions, which contain the same placement nodes for all considered loading profiles. Thus, there are significant evidences that it is possible to properly carry out the optimal placement of PQ monitors, assuming the distribution system modeled and simulated without loads.
\end{abstract}

\section{Key words}

Distribution systems, power quality, optimal allocation, load profile, voltage sags.

\section{Introduction}

Distribution Systems (DS), mainly those with overhead lines, constantly undergo different types of disturbances and failures that usually arises from several situations, such as adverse weather conditions, diverse short-circuit conditions, operational mistakes and equipment malfunctioning.

The occurrence of the aforementioned disturbances causes a non-ideal power supply condition, which may lead to the deterioration of the Power Quality (PQ). Among all possible disturbances in DS, short duration voltage variations, especially voltage sags, are the most frequent in DS and easily perceptible by customers [1], [2].

Normally, the most severe voltage sags may propagate throughout the whole system mainly when caused by low impedance short-circuits or even by the energization of high power loads.

Voltage sags are defined as short voltage variations with the remaining voltage magnitude between $0.1 \mathrm{p}$.u. and 0.9 p.u., lasting less than or equal to one minute [3].

In this context, voltage sags may be considered as a major cause of customer complaints, since they may cause malfunctioning of sensitive loads which leads to considerable financial losses due to the interruption of industrial processes [4].

Thereby, properly predicting the DS performance regarding the effects of voltage sags may become a complex task, which demands high amounts of measurements, data storage and processing. Moreover, to achieve such monitoring quality it would be required one monitor installed at each node of the DS, increasing the financial costs. Nevertheless, a long period of measurements would be crucial in order to compile a relevant and meaningful database of the DS behavior.

Recently, some researches have been proposed to overcome the aforementioned drawbacks, which include alternatives to deal with the prediction of the DS behavior considering the voltage sags effects and methods for optimized placement of monitors, aiming to reduce the financial costs regarding the PQ monitors' purchase and maintenance [5]-[11].

Despite the efficiency of the reported researches, only a few of them are especially targeted for DS. However, such researches only consider one loading profile of the studied DS when carrying out the monitor placement procedure. Thus, it is difficult to assure the effectiveness of the placement solution, or even infer about its behavior, when different loading conditions are applied in the DS. As the DS loading profile significantly varies along the day, it is necessary to study the influence of those distinct loading conditions in the optimized placement of PQ monitors. 
Considering the aforementioned context, this research proposes the performance assessment of a branch and bound based methodology for the optimal placement of PQ monitors considering different loading scenarios. The analyzed scenarios were obtained by considering different loading on the standard IEEE 34 nodes test feeder [12].

The use of the proposed methodology will provide important information about the voltage sags magnitudes, showing its influence and propagation behavior throughout all nodes of the system.

The branch and bound based algorithm will calculate the optimal number of PQ monitors, as well as its installation nodes, in order to achieve the complete monitoring of the DS regarding the voltage sags. Moreover, the placement solution will ensure that any voltage sag will be detected by at least one voltage monitor within the DS.

As the branch and bound based algorithm may provide equally feasible placement solutions, which contains different allocation nodes, an observability index will be determined. Thus, it will be possible to prioritize the installation of monitors at those nodes which allow the highest observability, so that the monitors will be more sensitized by the voltage sags.

\section{Methodology}

\section{A. DS modeling using ATP}

Traditionally, most part of the available measurements in DS are acquired at the system power substation, whereas none of the electrical quantities are monitored along the feeder itself [13]. Therefore, many disturbances that occur along the DS feeder and deteriorate the PQ are neither monitored nor assessed by the power company. Moreover, it is known that the DS loading changes throughout the day [13]. However, due to the lack of monitors assigned to register the dynamic and random behavior of loads, the exact characterization of the DS loading for detailed studies is hard to be obtained.

As early mentioned, the researches presented in [5]-[11] suggest important results of the optimized placement of the PQ monitors. Still, more detailed studies about the influences of the loading variations on the optimized placement of PQ monitors are needed. Thereby, considering four different loading profiles in a DS, the methodology presented in this paper aims to assess whether the effectiveness of the PQ monitors placement is directly affected or not.

The analyzed DS is the IEEE 34 nodes test feeder [12], which is shown in Fig. 1. This DS was modeled using the ATP (Alternative Transients Program) via ATPDraw interface [14]. In this study all DS loads were modeled as constant impedance and calculated based on the magnitudes of power and voltage available in [12].

The main feeder of this DS has its nominal voltage at $24.9 \mathrm{kV}$ and it is $57.6 \mathrm{~km}$ long. The nominal loading is approximately $2.05 \mathrm{MVA}$.

In order to keep the voltage profile within an acceptable range, this DS contains two voltage regulators and two shunt capacitor banks. Furthermore, it is important to mention that there are fixed and distributed loads, which are unbalanced. Besides, nodes 27 and 28 are the only ones in the system with its nominal voltage at $4.16 \mathrm{kV}$.

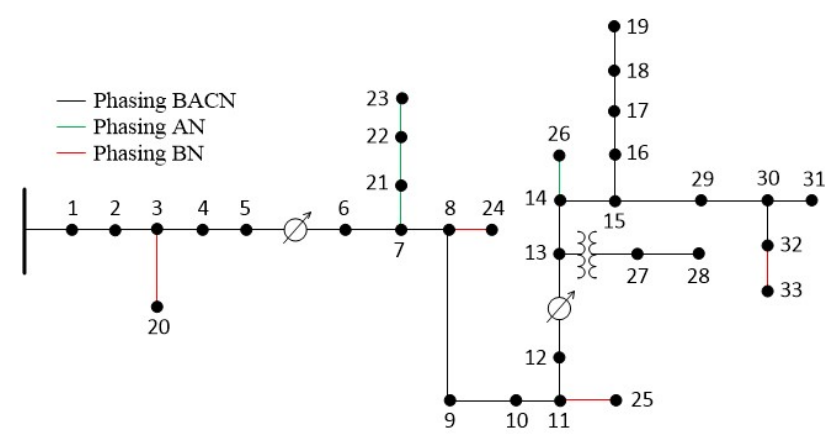

Fig.1. IEEE 34 nodes distribution test feeder.

The four loading scenarios are briefly described in the following.

The first considered scenario is the "Regular Loading", which consists of the normal loading provided in [12]. The "Heavy Loading" is the second scenario, and it contains a $20 \%$ raise from the regular condition of both active and reactive power of each load of the system, which totals 2.5 MVA for the system load as a whole. The "Light Loading" is the third considered scenario, and it contains a $20 \%$ reduction from the regular condition of both active and reactive power of each load of the system, which totals 1.65 MVA for the system load as a whole.

For the three aforementioned loading scenarios the taps of the voltage regulators where fixed.

Finally, the fourth scenario, called "No Loading", consists on the classical approach for short-circuits studies, i.e., the loads, capacitor banks and voltage regulators are neglected in the simulation analysis [13]. This loading scenario is an alternative approximation for those studies that require short-circuit simulations, and when precise information about the DS is unknown.

The phase "a" voltages for each node of the DS, corresponding to the four loading profiles, are shown in Fig. 2. Based on this figure, it is possible to observe that the light loading scenario has the highest pre-fault voltages, due to a smaller loading that causes less voltage drops along the feeder. The opposite situation is observed for the heavy loading scenario, which has the lowest steady-state pre-fault voltages, as the loading magnitude is higher.

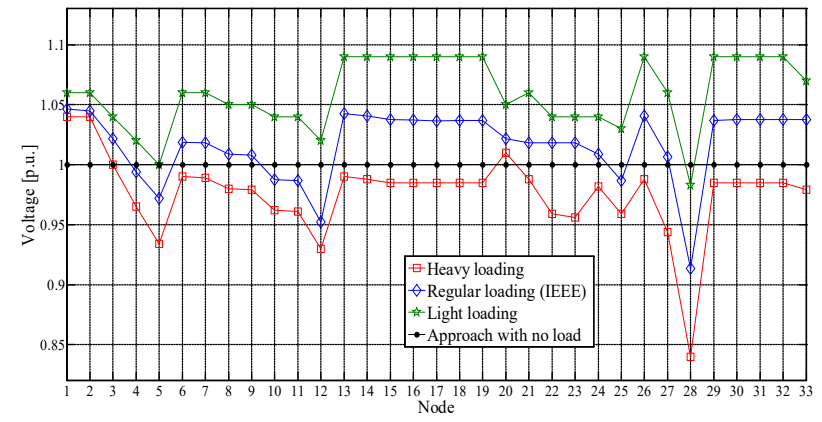

Fig.2. Phase "a" voltage profiles for all loading scenarios.

Because it would be technically difficult and impracticable to represent all possible loading combinations of the DS, the extreme loading conditions (heavy and light) are analyzed together with the regular loading. With this approach, it is intended to cover all other possible operation point of the DS, i.e., the other 
loading combinations would be adequately represented within the heavy and light loadings.

Thereafter, the proposed methodology consisted on applying single phase short-circuits at all nodes of the test DS. In this research, single phase short-circuits at phase "a" were studied, and the remaining voltages during the short-circuit simulations were measured to build the During Fault Voltage Matrix (DFVM) [11]. Each column of the DFVM represents the node where the fault was applied, while the rows contain the remaining voltage magnitudes at each node of the DS due to the respective fault application. The single phase short-circuit was studied due to its major probability of occurrence in overhead DS [13].

It is important to mention that single phase short-circuits at phase "b" were simulated at nodes 20, 24, 25 and 33 of the test DS, since they do not involve the phase "a" of the system. Thus, in such cases the voltages at phase "b" of all the other nodes of the DS were monitored.

Moreover, this research proposes the single phase shortcircuit analysis with fault resistances equal to 0 (zero) and $40 \Omega$. Therefore, for each one of the four loading scenarios, 33 short-circuit simulations will be carried out including the two fault resistances, totaling 264 short-circuit simulations within the DS.

The DFVM is calculated for the three phases of the system, storing all remaining voltages at each node due to the aforementioned short-circuits simulations. Thus, 24 DFVM are obtained, which after a phase aggregation procedure will result in eight voltage sag matrices. These voltage sag matrices are later used as inputs of the PQ monitor placement algorithm, as described in the following.

\section{B. Optimization problem}

It is known from practical situations that the complete monitoring of the DS may represent the purchase and maintenance of a large amount of PQ monitors, which leads to expensive investments in the monitoring planning. Therefore, the optimization problem aims to determine the minimal number of PQ monitors that are necessary to monitor voltage sags. Moreover, the optimization process will provide the nodes where the PQ monitors should be installed in order to maximize the voltage sags detection within the considered DS.

In order to quantify the monitoring reach of one PQ monitor installed at a certain node in the DS, a voltage threshold value $(\tau)$ is set after the DFVM calculation. This voltage threshold remains between 0.9 p.u. and 0.1 p.u., and defines the minimal remaining voltage that will sensitize the PQ monitors. The combination of the DFVM and $\tau$ will result in the Binary Observability Matrix (BOM). Thus, the elements of the BOM are filled with 0 (zero), if the magnitude of the measured remaining voltage is greater than $\tau$, and filled with 1 (one) if it is smaller or equal to $\tau$.

The branch and bound algorithm was used in order to determine the best nodes that the PQ monitors should be installed to ensure the maximum observability of the DS. The branch and bound is an exact algorithm for implicit enumeration, and its structure is based on building a linear programming tree that explores the possible solutions in the search space [15].

The objective function is a minimization function (1) that aims to minimize the sum of the elements of the monitoring vector $(X)$, associating the priority of installation $(P)$ of the PQ monitors in accordance with the higher voltage sag observability.

$$
\min f(x)=\sum_{i=1}^{n} x_{i} p_{i}=X \cdot P^{t}
$$

The number of nodes $(n)$ in the DS defines the dimension of the monitoring vector $X(n \times 1)$, which is a binary vector that provides the solution for the optimized placement of PQ monitors. This binary vector contains the minimal number of monitoring equipment that are needed to detect the possible faults within the DS, as well as the exact nodes one should install them. Therefore, during the optimization process the binary vector $X$ is filled out with 0 (zero) or 1 (one), respectively representing whether the monitor is installed or not.

In (1), the priority vector $P$ is a coefficient vector of $X$, which has a $1 \times n$ dimension. A priority coefficient is assigned for all the elements of $P$, representing the monitor installation priority in the optimization process. Thus, to completely determine $P$, an Observability Index (OI) of the measurements is calculated for each available installation point of a PQ monitor. The OI provides the average amount of the monitored voltage sags at each node, as shown in (2).

$$
O I=\frac{\sum_{i=1, j=1}^{n} \text { bom }_{i j} \cdot x_{i}}{n} \quad x_{i}=\left\{\begin{array}{l}
1, \text { if } i=j \\
0, \text { otherwise }
\end{array}\right.
$$

As the OI is obtained for all available allocation points of the PQ monitors, it is possible to define the nodes that must be prioritized in the optimization process. This priority definition is finally done by assigning the lower priority coefficient (with respect to the other elements of vector $P$ ) to those nodes that possess the highest observability. Thus the element positions of vector $P$ (priority coefficients) are sorted in an increasing way, i.e., from the highest to the lowest observability.

The lowest integer coefficients will be prioritized in the search of the optimal solution. Therefore, if there is one feasible solution that contains the penalized variable, the algorithm will calculate the optimal solution, prioritizing the monitor allocation at those nodes with the lowest coefficients, i.e., those nodes that possess the highest observability.

After assigning the weight for each element of $P$, it is possible to observe the system as a whole employing the allocation solution of the PQ monitors, as it aims to achieve the best placement of a reduced amount of PQ monitors.

Despite the use of the branch and bound algorithm in this research, it is possible to easily adapt the proposed methodology if the computational complexity of the studied problem increases. Alternatively, in order to carry 
out the optimization process, heuristics methods that are suitable for the described binary logic can be used.

The problem constraints were formulated in order to ensure detection of any voltage sag within the DS by at least one of the installed PQ monitors. This condition is set when the vector product between the transposed BOM and vector $X$ is greater than or equal to 1 (one), as shown in (3).

$$
\text { Subject to: } \sum_{i=1}^{n} \text { bom }_{i j} x_{i} \geq 1
$$

Thereby, for each loading profile and respective fault resistances, the placement algorithm calculates an optimal solution, considering each chosen voltage threshold, prioritizing the PQ monitors installation at the nodes with the highest observability.

\section{Results}

As mentioned in section 2- $A$, in order to assess the performance of the proposed methodology, the IEEE 34 nodes test feeder was modeled considering four loading scenarios (Fig. 2) and two fault resistances (0 $\Omega$ and $40 \Omega$ ) for the short-circuit simulations.

For each fault resistance four voltage sag matrices were obtained, i.e., one matrix for each loading scenario with the respective fault resistances. Thereafter, as described in section 2- $B$, it was necessary to define the voltage threshold. Usually, this definition is not an easy task, because a tradeoff between the monitor sensibility and the redundancy of the measures is needed. The highest the voltage threshold is, the more sensitized the monitors will be by the voltage sags occurrence. However, it will reduce the redundancy of the measures, since a lower number of PQ monitors is required. This fact may compromise the monitoring reliability and even overload the storage capacity of the monitor.

In this context the threshold setting was based in the probability occurrence of the voltage sags presented in [16]. In this study it is reported that almost $90 \%$ of the voltage sags have its remaining RMS voltage between $50 \%$ to $85 \%$. Therefore, in this research the thresholds $\tau$ considered will be $0.4,0.5,0.6,0.7,0.8$ and 0.9 p.u., resulting in $6 \mathrm{BOM}$ for each voltage sag matrix. Thus, computing the 8 voltage sags matrices calculated in the short-circuit simulations using the ATP, 48 BOM are build.

For each one of the $48 \mathrm{BOM}$, the branch and bound algorithm was executed in order to calculate the minimal number and the correct location of the PQ monitors. However, at each run of the algorithm a different feasible allocation solution was obtained, since there is more than one solution available, resulting in different allocation points. Thus, it was not possible to perform an adequate comparison among the different allocation solution when considering distinct loading scenarios.

To overcome this drawback and obtain a solution that could cover all considered loading scenarios, as well as both fault resistances ( $0 \Omega$ and $40 \Omega$ ), the OI was calculated, as described in section 2- $B$.

Tables I and II show the OI calculated by (2), for all loading scenarios and fault resistance of $0 \Omega$.
Table I. - OI for "No Loading" and "Regular Loading" scenarios, considering $0 \Omega$ fault resistance.

\begin{tabular}{|c|c|c||c|c|c|}
\hline Node & $\begin{array}{c}\text { No } \\
\text { loading }\end{array}$ & $\begin{array}{c}\text { Regular } \\
\text { loading }\end{array}$ & Node & $\begin{array}{c}\text { No } \\
\text { loading }\end{array}$ & $\begin{array}{c}\text { Regular } \\
\text { loading }\end{array}$ \\
\hline \hline $\mathbf{1}$ & 2.2727 & 2.0000 & $\mathbf{1 8}$ & 8.4242 & 8.3939 \\
\hline $\mathbf{2}$ & 2.4242 & 2.0303 & $\mathbf{1 9}$ & 8.4242 & 8.3939 \\
\hline $\mathbf{3}$ & 4.1515 & 3.6667 & $\mathbf{2 0}$ & 4.1818 & 3.6970 \\
\hline $\mathbf{4}$ & 5.5758 & 5.3636 & $\mathbf{2 1}$ & 6.5152 & 6.4545 \\
\hline $\mathbf{5}$ & 6.5152 & 6.5758 & $\mathbf{2 2}$ & 6.7273 & 6.6970 \\
\hline $\mathbf{6}$ & 6.5152 & 6.4545 & $\mathbf{2 3}$ & 6.7273 & 6.6970 \\
\hline $\mathbf{7}$ & 6.5152 & 6.4545 & $\mathbf{2 4}$ & 7.0000 & 6.9091 \\
\hline $\mathbf{8}$ & 7.0000 & 6.9091 & $\mathbf{2 5}$ & 7.6061 & 7.5455 \\
\hline $\mathbf{9}$ & 7.0000 & 6.9091 & $\mathbf{2 6}$ & 8.4242 & 8.3939 \\
\hline $\mathbf{1 0}$ & 7.5152 & 7.5152 & $\mathbf{2 7}$ & 8.5758 & 8.5455 \\
\hline $\mathbf{1 1}$ & 7.5758 & 7.5152 & $\mathbf{2 8}$ & 8.7273 & 8.6667 \\
\hline $\mathbf{1 2}$ & 8.3939 & 8.4242 & $\mathbf{2 9}$ & 8.4242 & 8.3939 \\
\hline $\mathbf{1 3}$ & 8.4242 & 8.3636 & $\mathbf{3 0}$ & 8.4242 & 8.3939 \\
\hline $\mathbf{1 4}$ & 8.4242 & 8.3939 & $\mathbf{3 1}$ & 8.4242 & 8.3939 \\
\hline $\mathbf{1 5}$ & 8.4242 & 8.3939 & $\mathbf{3 2}$ & 8.4242 & 8.3939 \\
\hline $\mathbf{1 6}$ & 8.4242 & 8.3939 & $\mathbf{3 3}$ & 8.4242 & 8.3939 \\
\hline $\mathbf{1 7}$ & 8.4242 & 8.3939 & & & \\
\hline
\end{tabular}

Table II. - OI for "Light Loading" and "Heavy Loading" scenarios, considering $0 \Omega$ fault resistance.

\begin{tabular}{|c|c|c||c|c|c|}
\hline Node & $\begin{array}{c}\text { Light } \\
\text { loading }\end{array}$ & $\begin{array}{c}\text { Heavy } \\
\text { loading }\end{array}$ & Node & $\begin{array}{c}\text { Light } \\
\text { loading }\end{array}$ & $\begin{array}{c}\text { Heavy } \\
\text { loading }\end{array}$ \\
\hline \hline $\mathbf{1}$ & 2.0000 & 2.0000 & $\mathbf{1 8}$ & 8.3939 & 8.3939 \\
\hline $\mathbf{2}$ & 2.0303 & 2.0303 & $\mathbf{1 9}$ & 8.3939 & 8.3939 \\
\hline $\mathbf{3}$ & 3.6364 & 3.6970 & $\mathbf{2 0}$ & 3.6667 & 3.7273 \\
\hline $\mathbf{4}$ & 5.3333 & 5.3636 & $\mathbf{2 1}$ & 6.4545 & 6.4545 \\
\hline $\mathbf{5}$ & 6.5455 & 6.6061 & $\mathbf{2 2}$ & 6.6667 & 6.7576 \\
\hline $\mathbf{6}$ & 6.4545 & 6.4545 & $\mathbf{2 3}$ & 6.6970 & 6.7576 \\
\hline $\mathbf{7}$ & 6.4545 & 6.4545 & $\mathbf{2 4}$ & 6.9091 & 6.9091 \\
\hline $\mathbf{8}$ & 6.9091 & 6.9091 & $\mathbf{2 5}$ & 7.5455 & 7.5455 \\
\hline $\mathbf{9}$ & 6.9091 & 6.9394 & $\mathbf{2 6}$ & 8.3939 & 8.3939 \\
\hline $\mathbf{1 0}$ & 7.5152 & 7.5152 & $\mathbf{2 7}$ & 8.5758 & 8.5758 \\
\hline $\mathbf{1 1}$ & 7.5152 & 7.5152 & $\mathbf{2 8}$ & 8.6970 & 8.6970 \\
\hline $\mathbf{1 2}$ & 8.3939 & 8.4545 & $\mathbf{2 9}$ & 8.3939 & 8.3939 \\
\hline $\mathbf{1 3}$ & 8.3636 & 8.3636 & $\mathbf{3 0}$ & 8.3939 & 8.3939 \\
\hline $\mathbf{1 4}$ & 8.3939 & 8.3939 & $\mathbf{3 1}$ & 8.3939 & 8.3939 \\
\hline $\mathbf{1 5}$ & 8.3939 & 8.3939 & $\mathbf{3 2}$ & 8.3939 & 8.3939 \\
\hline $\mathbf{1 6}$ & 8.3939 & 8.3939 & $\mathbf{3 3}$ & 8.3939 & 8.3939 \\
\hline $\mathbf{1 7}$ & 8.3939 & 8.3939 & & & \\
\hline
\end{tabular}

As also detailed in section 2- $B$, the allocation algorithm prioritizes the installation of the PQ monitors at those nodes with the highest OI, assigning to them a lower index of the respective $P$ element when compared with the nodes that are less sensitized by the voltage sags. Thereby, for the OI shown in Table I and II, the priority vector $P$ is calculated as registered in Table III.

Table III. - PQ monitor installation priority for all loading scenarios, considering $0 \Omega$ fault resistance.

\begin{tabular}{|c|c|c|c|c|c|c|c|c|}
\hline & \multicolumn{8}{|c|}{ Installation Priority } \\
\hline Load & \begin{tabular}{l|l|}
$(1)$ & $(2)$ \\
\end{tabular} & (3) ...(12) & (13) & (14)...(22) & (23)...(26) & (27) & & (29)... (33) \\
\hline No load & \begin{tabular}{|l|l|}
15 & 14 \\
\end{tabular} & $13 \ldots 4$ & 3 & $\begin{array}{lll}3 \ldots 9 \\
\end{array}$ & $9 \ldots 3$ & 2 & 1 & $3 \ldots 3$ \\
\hline Light & \begin{tabular}{l|l|}
16 & 15 \\
\end{tabular} & $14 \ldots 3$ & 4 & $\begin{array}{lll}3 \ldots 9 \\
\end{array}$ & $8 .$. & 2 & 1 & 3. \\
\hline Regular & \begin{tabular}{|l|l|}
17 & 16 \\
\end{tabular} & $15 \ldots 3$ & 5 & $\begin{array}{l}4 \ldots 9 \\
\end{array}$ & $10 \ldots 4$ & 2 & 1 & $4 \ldots 4$ \\
\hline Heavy & \begin{tabular}{|l|l|}
17 & 16 \\
\end{tabular} & $15 \ldots 3$ & 5 & $\begin{array}{lll}4 & \ldots & 10\end{array}$ & $10 \ldots 4$ & 2 & 1 & $4 \ldots 4$ \\
\hline
\end{tabular}


As it is possible to verify in Table III, node 28 has the highest priority for all the loading scenarios considered. This node was the most sensitized by the voltage sags for the four analyzed loading scenarios. It does not necessarily mean that node 28 is the optimal point for the monitor installation, since this decision is made by the branch and bound algorithm, i.e., if node 28 is included in the possible solution set it will be chosen as one of the best place to install the PQ monitor.

After the definition of vector $P$, the branch and bound algorithm was executed, and the nodes where the monitors should be installed, as well as the number of monitors required to monitor each threshold voltage, are shown in Tables IV and V. As node 28 was found as belonging to the feasible solution set when considering all analyzed scenarios, this node was then prioritized for the PQ monitor installation. These results provided an adequate comparison of the allocation solutions calculated by the optimization algorithm. Thus it is possible to state that even changing the loading of the DS by $\pm 20 \%$ from its regular loading, the number of PQ monitors remained the same for the voltage threshold from 0.9 p.u. to 0.5 p.u..

For the 0.4 p.u. threshold and the "Light Loading" scenario, the algorithm provided the placement of the PQ monitor at node 23 instead at node 22 , as node 23 is prioritized in search of the allocation solution (Table III). However, either node 22 or node 23 leads to optimal feasible solutions for all loading scenarios, as it is possible to verify at Table VI.

It is also possible to observe in Table IV that considering the 0.4 p.u. threshold for "Heavy Loading" scenario, only one monitor was needed to detect any voltage sag within de DS, whereas two monitors were needed for all other scenarios. This can be explained since the "Heavy Loading" scenario contains the lowest pre-fault voltage values (Fig. 2), which consequently causes the most severe voltage sags after short-circuits are applied.

Table IV. - Optimal placement of PQ monitors for single phase short-circuits ( $0 \Omega$ fault resistance).

\begin{tabular}{|l|c|c|c|c|}
\hline \multirow{2}{*}{$\tau$ (p.u.) } & \multicolumn{4}{|c|}{ Required monitors/ \{Monitoring node\} } \\
\cline { 2 - 5 } & $\begin{array}{c}\text { No } \\
\text { loading }\end{array}$ & $\begin{array}{c}\text { Light } \\
\text { loading }\end{array}$ & $\begin{array}{c}\text { Regular } \\
\text { loading }\end{array}$ & $\begin{array}{c}\text { Heavy } \\
\text { loading }\end{array}$ \\
\hline \hline$\leq 0.9$ & $1 /\{28\}$ & $1 /\{28\}$ & $1 /\{28\}$ & $1 /\{28\}$ \\
\hline$\leq 0.8$ & $1 /\{28\}$ & $1 /\{28\}$ & $1 /\{28\}$ & $1 /\{28\}$ \\
\hline$\leq 0.7$ & $1 /\{28\}$ & $1 /\{28\}$ & $1 /\{28\}$ & $1 /\{28\}$ \\
\hline$\leq 0.6$ & $1 /\{28\}$ & $1 /\{28\}$ & $1 /\{28\}$ & $1 /\{28\}$ \\
\hline$\leq 0.5$ & $1 /\{28\}$ & $1 /\{28\}$ & $1 /\{28\}$ & $1 /\{28\}$ \\
\hline$\leq 0.4$ & $2 /\{22 ; 28\}$ & $2 /\{23 ; 28\}$ & $2 /\{22 ; 28\}$ & $1 /\{28\}$ \\
\hline
\end{tabular}

Table $\mathrm{V}$ presents the results obtained for all loading scenarios when single phase short-circuits with $40 \Omega$ fault resistance are considered. Despite the algorithm was executed for all voltage threshold, it was observed that the higher the fault resistance is, the less severe are the voltage sags magnitude. Thus, allocation solutions were only obtained for the highest voltage thresholds.

The placement of PQ monitors (M1 and M2) for the 34 nodes DS is depicted in Fig. 3. In this figure it is highlighted that only one PQ monitor installed at node 28 would be enough to monitor any voltage sag due to single phase short-circuits. Moreover, it is possible to state that this allocation solution is suitable even if loading variations are verified. Thus, it is demonstrated that even though the load information are neglected when modeling the DS, usually due to the lack of precise data, the DS monitoring will not be affected.

Table V. - Optimal placement of PQ monitors for single phase short-circuits ( $40 \Omega$ fault resistance).

\begin{tabular}{|c|c|c|c|c|}
\hline \multirow{2}{*}{$\tau$ (p.u.) } & \multicolumn{4}{|c|}{ Required monitors/ \{Monitoring node\} } \\
\cline { 2 - 5 } & $\begin{array}{c}\text { No } \\
\text { loading }\end{array}$ & $\begin{array}{c}\text { Light } \\
\text { loading }\end{array}$ & $\begin{array}{c}\text { Regular } \\
\text { loading }\end{array}$ & $\begin{array}{c}\text { Heavy } \\
\text { loading }\end{array}$ \\
\hline \hline$\leq 0.9$ & $1 /\{28\}$ & $1 /\{28\}$ & $1 /\{28\}$ & $1 /\{28\}$ \\
\hline$\leq 0.8$ & - & $1 /\{28\}$ & $1 /\{28\}$ & $1 /\{28\}$ \\
\hline$\leq 0.7$ & - & - & - & $1 /\{28\}$ \\
\hline
\end{tabular}

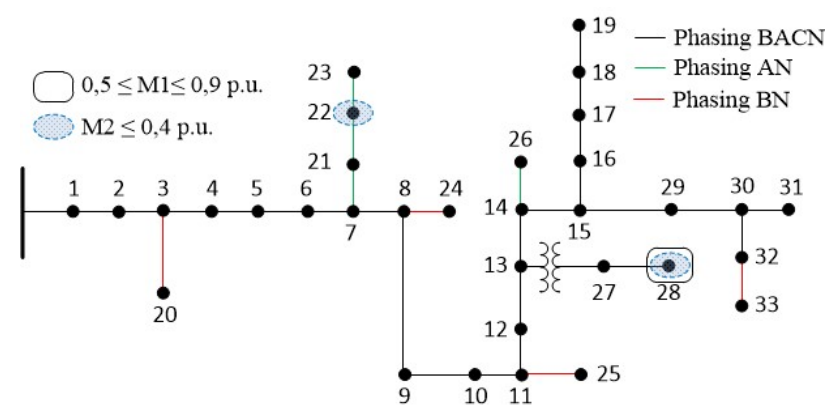

Fig. 3. Optimal placement of PQ monitors M1 and M2 at IEEE 34 nodes test feeder considering different loading scenarios.

Completing the analysis of Fig. 3, it is shown that if the voltage threshold is reduced, there is more than one option to install the PQ monitors, which could provide a higher redundancy of the measures. Therefore, if more PQ monitors are available, or if the power company's budget is adequate for purchasing new monitors, it could raise the DS monitoring efficiency and reliability.

Solutions should be presented in a way that allows the DS operator to decide about the best monitor allocation set. In this context, Tables VI and VII contain other possible solutions that are suitable for all studied loading scenarios. Results are displayed following the installation priority order of the PQ monitors, so that is possible to verify what are the nodes that possess the higher capacity of monitoring the voltage sags. Furthermore, the total number of possible solutions for each voltage threshold is also shown in Table VI and VII.

Table VI. - Other possible solutions suitable for all loading scenarios following the installation priority order $(0 \Omega$ fault resistance).

\begin{tabular}{|l|c|c|}
\hline $\boldsymbol{\tau}$ (p.u.) & $\begin{array}{c}\text { Solutions } \\
\text { number }\end{array}$ & Nodes for monitor installation \\
\hline \hline$\leq \mathbf{0 . 9}$ & 29 & $\begin{array}{c}\{28\},\{27\},\{12\},\{14\},\{17\},\{19\},\{26\}, \\
\{29\},\{31\},\{33\},\{13\},\{25\}, \ldots,\{4\}\end{array}$ \\
\hline$\leq \mathbf{0 . 8}$ & 19 & $\begin{array}{c}\{28\},\{27\},\{12\},\{14\},\{17\},\{19\},\{26\}, \\
\{29\},\{31\},\{33\},\{13\},\{25\}, \ldots,\{10\}\end{array}$ \\
\hline$\leq \mathbf{0 . 7}$ & 2 & $\{28\},\{27\}$ \\
\hline$\leq \mathbf{0 . 6}$ & 2 & $\{28\},\{27\}$ \\
\hline$\leq \mathbf{0 . 5}$ & 1 & $\{28\}$ \\
\hline$\leq \mathbf{0 . 4}$ & 2 & $\{22 ; 28\},\{23 ; 28\}$ \\
\hline
\end{tabular}


Table VII. - Other possible solutions suitable for all loading scenarios following the installation priority order ( $40 \Omega$ fault resistance).

\begin{tabular}{|c|c|c|c|c|}
\hline \multirow[b]{2}{*}{$\begin{array}{c}\tau \\
(\text { p.u. })\end{array}$} & \multicolumn{4}{|c|}{ Nodes for monitor installation } \\
\hline & $\begin{array}{l}\text { No } \\
\text { load }\end{array}$ & $\begin{array}{l}\text { Light } \\
\text { loading }\end{array}$ & $\begin{array}{l}\text { Regular } \\
\text { loading }\end{array}$ & Heavy loading \\
\hline$\leq 0.7$ & - & - & - & $\{28\}$ \\
\hline$\leq \mathbf{0 . 8}$ & - & $\{28\}$ & $\begin{array}{c}\{28\},\{12\}, \\
\{5\}\end{array}$ & $\begin{array}{c}\{28\},\{27\},\{12\},\{25\} \\
\{10\},\{11\},\{22\},\{23\},\{5\}\end{array}$ \\
\hline$\leq \mathbf{0 . 9}$ & $\begin{array}{r}\{28 \\
\{29\},\end{array}$ & $\begin{array}{r}27\},\{12 \\
9\},\{31\}, \\
\{8\},\{2\end{array}$ & $\begin{array}{l}\{14\},\{15\},\{ \\
32\},\{33\},\{1 \\
\},\{23\},\{22\}\end{array}$ & $\begin{array}{l}6\},\{17\},\{18\},\{19\},\{26\}, \\
\},\{25\},\{11\},\{10\},\{5\},\{9\}, \\
\{21\},\{6\},\{7\},\{4\}\end{array}$ \\
\hline
\end{tabular}

\section{Conclusion}

The placement methodology of PQ monitors presented in this research was able to indicate the best installation points for monitors, ensuring the voltage sag detection for a wide operation scenario that the DS may experience.

The allocation solutions provided by the proposed methodology covers distinct short-circuit simulations evaluated at all DS nodes, including variations of the fault resistance ( $0 \Omega$ and $40 \Omega$ ). Furthermore, those short-circuit simulations were carried out including four different loading levels of the test DS. This consideration was done in order assess whether the effectiveness of the PQ monitors placement is directly affected or not.

Results show that based on the calculation of the OI, the allocation algorithm was able to prioritize the installation of the PQ monitors at those nodes that are more sensitized by the voltage sags. By this approach, it is possible to state that the installation of only one PQ monitor at node 28 of the test DS is enough to detect the voltage sags caused by the single phase short-circuits. Furthermore, this allocation solution is suitable even when the DS loading changes ( $\pm 20 \%$ from the regular loading), considering the voltage threshold is set from 0.5 p.u. to 0.9 p.u..

Results also demonstrate that if the voltage threshold is set to be lower or equal to 0.4 p.u., more than one possible allocation solution including node 28 is available. Setting $\tau \leq 0.4$ p.u., will raise the redundancy of the measures, but will also demand a larger number of PQ monitors.

It is important to highlight that the convergence of the allocation solutions calculated by the proposed methodology, considering all the studied loading scenarios, shows that is possible to execute the placement of PQ monitors considering the DS modeled without loads ("No Loading" scenario). Indeed, as there are several drawbacks in obtaining precise information about all loads of a real DS, in order to perform its computational modeling, the "No Loading" scenario presents itself as a viable and suitable alternative for carrying out the studies of the optimized placement of PQ monitors in DS.

\section{Acknowledgement}

The authors gratefully acknowledge the Department of Electrical and Computer Engineering, São Carlos School of Engineering, University of São Paulo (Brazil), and the financial support provided by FAPESP (Fundação de
Amparo à Pesquisa do Estado de São Paulo) and Eletrobras Distribuição Piauí - EDPI (PD-0042/2014ANEEL).

\section{References}

[1] M. F. Mcranaghan, D. R. Mueller and M. J. Samotyj, "Voltage Sags in Industrial Systems," Industry Applications, IEEE Transactions on, vol.29, no.2, pp.397403, Mar/Apr 1993.

[2] M. H. J. Bollen, M. R Qader and R. N. Allan, "Stochastical and statistical assessment of voltage dips," Tools and Techniques for Dealing with Uncertainty (Digest No. 1998/200), IEEE Colloquium on, pp.5/1-5/4, 27 Jan 1998.

[3] IEEE Recommended Practice for Monitoring Electric Power Quality, IEEE Standard 1159 - 1995, Nov. 1995.

[4] M. H. J. Bollen, "Voltage sag: Effects, Mitigation and Prediction," Power Engineering Journal, vol.10, no.3, pp.129-135, June 1996.

[5] G. Olguin, F. Vuinovich and M. H. J. Bollen, "An optimal monitoring program for obtaining Voltage sag system indexes," Power Systems, IEEE Transactions on , vol.21, no.1, pp. 378- 384, Feb. 2006.

[6] D. C. S. Reis, P. R. C. Villela, C. A. Duque and P. F. Ribeiro, "Transmission Systems Power Quality Monitors Allocation," Power and Energy Society General Meeting Conversion and Delivery of Electrical Energy in the 21st Century, 2008 IEEE , pp.1-7, 20-24, July 2008.

[7] E. Espinosa-Juárez, A. Hernandes and G. Olguin, "An Approach Based on Analytical Expressions for Optimal Location of Voltage Sags Monitors," Power Delivery, IEEE Transactions on, vol.24, no.4, pp.2034-2042, Oct. 2009.

[8] C. F. M. Almeida and N. Kagan, "Allocation of Power Quality Monitors by Genetic Algorithms and Fuzzy Sets Theory," Intelligent System Applications to Power Systems, 2009. ISAP '09. 15th International Conference on, pp.1,6, 8-12, Nov. 2009.

[9] F. Salim and K. M. Nor, "Voltage sags observation through optimal monitor locations," In Harmonics and Quality of Power (ICHQP), 14th International Conference on, 2010.

[10] M. Oleskovicz, H. M. G. C. Branco, R. P. M. da Silva, D.V. Coury and A. C. B. Delbem, "A Compact Genetic Algorithm structure used for the optimum allocation of power quality monitors based on electrical circuit topology," in Harmonics and Quality of Power (ICHQP), 2012 IEEE 15th International Conference on, pp.34-39, 17-20, June 2012.

[11] T. R. Kempner, A. Q. Santos and M. Oleskovicz, "Optimized monitoring of voltage sags in distribution systems caused by balanced and unbalanced shortcircuits," in PES General Meeting | Conference \& Exposition, 2014 IEEE , pp.1-5, 27-31, July 2014.

[12] IEEE PES Test Feeders, "Distribution System Analysis Subcommittee," IEEE Power Engineering Society, Available:

http://www.ewh.ieee.org/soc/pes/dsacom/testfeeders.html

[13] W. H. Kersting, Distribution System Modeling and Analysis. 2nd ed. Boca Raton, Florida USA. CRC Press LLC. 421 p., 2007.

[14] ATPDraw, "Help and about ATPDraw," Avaliable: http://www.atpdraw.net/help.php.

[15] L. A. Wolsey, Integer Programming, John Wiley \& Sons, ISBN: $9780471283669,1998$.

[16] EPRI - Electric Power Research Institute. Distribution System Power Quality Assessment: Phase II. Voltage Sag and Interruption Analysis. Project Manager A. Sundaram, pp. 5-17, 2003. 